\title{
Effects of Rotation Rate and Seasonal Forcing on the ITCZ Extent in Planetary Atmospheres
}

\author{
SEAN FAULK \\ Department of Earth, Planetary, and Space Sciences, University of California, Los Angeles, Los Angeles, California \\ JONATHAN MiTCHELL \\ Department of Earth, Planetary, and Space Sciences, and Atmospheric and Oceanic Sciences, University of California, \\ Los Angeles, Los Angeles, California \\ SIMONA BORDONI \\ California Institute of Technology, Pasadena, California
}

(Manuscript received 6 January 2016, in final form 14 November 2016)

\begin{abstract}
The authors study a wide range of atmospheric circulations with an idealized moist general circulation model to evaluate the mechanisms controlling intertropical convergence zone (ITCZ) migrations. They employ a zonally symmetric aquaplanet slab ocean of fixed depth and force top-of-atmosphere insolation to remain fixed at the pole for an "eternal solstice" simulation and also vary seasonally for a range of rotation rates, keeping all other parameters Earth-like. For rotation rates $\Omega_{E} / 8$ and slower, a transient maximum in zonal-mean precipitation appears at the summer pole; however, the ITCZ associated with the ascending branch of the Hadley circulation lies at $\sim 60^{\circ}$. The authors assess how widely used predictors of the ITCZ position perform in this wide parameter space. Standard predictors based on different estimates of the Hadley cell's poleward extent are correlated with but overestimate off-equatorial ITCZ locations. Interestingly, in the eternal-solstice case for Earth's rotation rate, the ITCZ remains at subtropical latitudes even though the lower-level moist static energy maximizes at the summer pole. While seemingly at odds with convective quasiequilibrium arguments, this can happen because at Earth's rotation rates, the thermal stratification set in convective regions can only be communicated within the tropics, where temperature gradients are constrained to be weak. The authors therefore develop an understanding of the ITCZ's position based on top-ofatmosphere energetics and the boundary layer momentum budget and argue that friction and pressure gradient forces determine the region of maximum convergence, offering a modified dynamical perspective on the monsoon-like seasonal weather patterns of terrestrial planets.
\end{abstract}

\section{Introduction}

One of the most prominent features of Earth's largescale atmospheric circulation in low latitudes is the intertropical convergence zone (ITCZ) (Waliser and Gautier 1993). Associated with the ascending branch of the Hadley cell, the ITCZ is the region of moist uplift, and thus of high precipitation and deep convection, occurring where the low-level winds of the two cells converge. During summer, this convergence zone migrates off the equator into the summer hemisphere, up to $10^{\circ}$ in

Corresponding authore-mail: Sean Faulk, spfaulk@ucla.edu oceanic regions and occasionally as far as $30^{\circ}$ in the case of the Asian summer monsoon (Yihui and Chan 2005).

The mechanisms that control the ITCZ remain unclear. Ideally, convergence occurs over the warmest surface waters. Indeed, in the northeast Pacific Ocean, the ITCZ remains north of the equator throughout the entire year, coinciding largely with high sea surface temperatures (SSTs), which do not follow maximal insolation during northern winter (Janowiak et al. 1995). However, previous observations show that the ITCZ does not always coincide with the SST maximum and corresponding local sea level pressure minimum (Ramage 1974; Sadler 1975). In light of such observations, and given the importance of moisture transport 
to tropical communities, considerable attention has been paid in the literature toward identifying and understanding controls on the ITCZ (Waliser and Somerville 1994; Sobel and Neelin 2006; Schneider et al. 2014). Most of this work can be roughly divided into two theories for understanding the ITCZ: one based on thermodynamics and the other on momentum dynamics.

\section{a. Thermodynamic theory}

Thermodynamic theories posit that convergence and precipitation are determined by local vertical temperature and moisture profiles governed by the moisture budget and surface and radiative fluxes (Sobel and Neelin 2006). When used in conjunction with the "weak temperature gradient" (WTG) approximation (Sobel et al. 2001), some such theories successfully reconstruct tropical convergence and tend to predict maximum convergence and rainfall over the warmest surface temperatures (Neelin and Held 1987; Raymond 2000; Sobel and Bretherton 2000). When applied to convergence zones associated with zonally averaged overturning circulations that conserve angular momentum (Lindzen and Hou 1988) and coupled to quasiequilibrium theories of moist convection (Arakawa and Schubert 1974; Emanuel et al. 1994; Nie et al. 2010), such theories argue that the ITCZ, defined here and throughout this paper as the latitude of maximum zonal-mean precipitation, lies just equatorward of the maximum low-level moist static energy (MSE) (Emanuel 1995; Privé and Plumb 2007; Bordoni and Schneider 2008).

However, the ITCZ has also been shown to respond strongly to extratropical thermal forcings, with shifts of the ITCZ into (away from) a relatively warmed (cooled) hemisphere, indicating that forcings remote from precipitation maxima can influence the tropical circulation and convergence zones (Chiang and Bitz 2005; Broccoli et al. 2006). The vertically integrated atmospheric energy budget has been invoked to explain ITCZ shifts in response to remote forcing and has led to the development of another diagnostic, the energy flux equator (EFE), which is the latitude at which the zonal-mean MSE meridional flux vanishes (Kang et al. 2008). In recent studies focused on global energy transports, the EFE has been shown to be well correlated with the ITCZ's poleward excursion into the summer hemisphere, and the energetic framework in general has proved useful for understanding the ITCZ and its migrations on different time scales (Kang et al. 2008, 2009; Chiang and Friedman 2012; Frierson and Hwang 2012; Donohoe et al. 2013; Frierson et al. 2013; Bischoff and Schneider 2014;
Schneider et al. 2014; Bischoff and Schneider 2016; Shekhar and Boos 2016).

\section{b. Dynamic theory}

Dynamic theories use the boundary layer momentum budget to determine winds and hence convergence, arguing that convection is driven primarily by boundary layer (BL) momentum dynamics (Lindzen and Nigam 1987; Waliser and Somerville 1994; Tomas and Webster 1997; Pauluis 2004). Perhaps the most influential of these studies, Lindzen and Nigam (1987) showed that the pressure gradients associated with even the small surface temperature gradients of the tropics have a substantial impact on low-level flow and convergence. Their model, built on tropical SST gradients, adequately reproduces the observed tropical convergence, though the model's assumptions have been debated. Back and Bretherton (2009) address such concerns by testing and expanding upon Lindzen and Nigam (1987), showing that SST gradients in reanalysis data are indeed consistent with BL convergence, which in turn causes deep convection. Along a similar vein, Tomas and Webster (1997) found that in regions where the surface crossequatorial sea level pressure gradient is weak, convection tends to coincide with the maximum SST, but in regions with a substantial cross-equatorial pressure gradient convection tends to lie equatorward of the maximum SST, also suggesting the importance of temperature gradients, as opposed to maxima, in driving the flow. Subsequent studies develop a theory to determine the location of the ITCZ based on the cross-equatorial pressure gradient, which drives anticyclonic vorticity advection across the equator to render the system inertially unstable (Tomas and Webster 1997; Tomas et al. 1999; Toma and Webster 2010). Thus, convergence and divergence are shown to be separated at the latitude where the zonal-mean absolute vorticity is equal to zero. Additionally, the role of rotation in "forcing" the ITCZ toward the equator has been put forth by Chao (2000) and Chao and Chen (2001), wherein two "forces" critical to monsoon onset are described as in balance: one, associated with Earth's rotation, toward the equator, and the other toward the SST peak.

Classical axisymmetric theory for tropical circulation based on angular momentum conservation can also be classified as dynamical (Held and Hou 1980). Though not predictive of the ITCZ location, these arguments do constrain the poleward extent of the Hadley cell to depend on planetary parameters such as rotation rate and radius. Studies have also highlighted the influence of eddy momentum fluxes on the Hadley circulation by studying wave-mean flow interactions (Pfeffer 1981; Becker et al. 1997), the response of Earth's Hadley circulation to 
seasonal transitions (Walker and Schneider 2005; Bordoni and Schneider 2008; Schneider and Bordoni 2008; Merlis et al. 2013), and the extent of the annual-mean Hadley circulation over a range of planetary parameters (Walker and Schneider 2006; Levine and Schneider 2011). Indeed, it has been suggested that the annual-mean Hadley circulation width may be affected by extratropical baroclinic eddies that limit its poleward extent (Schneider 2006; Levine and Schneider 2015).

\section{c. Generalizing theories of tropical circulation}

In our above classification of theories, we acknowledge that neither group is exclusively either thermodynamical or dynamical. Convective quasi-equilibrium (CQE) theory, for example, is often used in conjunction with the WTG approximation in tropical circulation regimes. By this, it is assumed that gravity waves efficiently communicate convective heating throughout the domain and, thereby, maintain a mean state with vanishingly small horizontal temperature gradients in the free troposphere. By this process, convection self-organizes to the maximum of low-level MSE and suppresses it elsewhere. Because of the WTG assumption, CQE theories of the ITCZ might actually be considered a hybrid of thermodynamics and dynamics. Furthermore, classical axisymmetric theory relies on energy conservation constraints to arrive at a scaling for the Hadley cell width, so it too may be considered a thermodynamic-dynamic hybrid.

Thus, CQE theory for the location of convergence is broadly connected, and in some ways analogous, to analytical dynamical theory based on angular momentum conservation and top-of-atmosphere (TOA) energy balance (Held and Hou 1980; Lindzen and Hou 1988; Satoh 1994; Caballero et al. 2008) as well as analytical thermodynamic theory for the location of an overturning circulation in a radiative-convective atmosphere based on a critical subcloud moist entropy curvature (Plumb and Hou 1992; Emanuel 1995). These analytical theories predict the extent of the Hadley cell, which we note must be distinguished from the location of the ITCZ itself. Indeed, with foresight of our results, this distinction may be particularly important in the context of other terrestrial planets.

Regions of seasonal convergence and ascent associated with the Hadley cell analogous to Earth's ITCZ exist on other planetary bodies, namely Mars (Haberle et al. 1993; Lewis 2003) and Titan, where the ascent region migrates significantly off the equator during summer solstice and nearly reaches the summer pole (Mitchell et al. 2006; Bouchez and Brown 2005; Porco et al. 2005). Many parameter space studies using general circulation models (GCMs) (Williams 1988; Navarra and Boccaletti 2002; Walker and Schneider 2006;
Mitchell and Vallis 2010; Mitchell et al. 2014; Dias Pinto and Mitchell 2014; Kaspi and Showman 2015) show that slowly rotating planets exhibit expanded Hadley cells and effectively become "all tropics" planets similar in circulation structure to Titan (Mitchell et al. 2006), consistent with classical axisymmetric theory, which predicts expanding Hadley cell widths with decreasing rotation rate and/or radius (Held and Hou 1980).

But no parameter-space studies have been done using a moist GCM with a seasonal cycle. We claim such experiments deserve investigation since the large-scale circulation is known to be sensitive to the addition of a seasonal cycle, which induces a cross-equatorial circulation and upper-level easterlies in the tropics and can lead to seasonal shifts in the Hadley cell's dominant angular momentum budget (Bordoni and Schneider 2008; Schneider and Bordoni 2008). Additionally, moisture greatly impacts the zonally averaged largescale circulation owing to the effects of latent heat on static stability and meridional temperature gradients (Frierson et al. 2006; Frierson 2008). We aim here to determine how sensitive the large-scale circulation is to slower rotation rates in the presence of moisture and a seasonal cycle. Furthermore, much of the work discussed above is relevant for near-equatorial ITCZs, where the influence of rotation and free-tropospheric temperature gradients are generally weak, but it thus remains unclear how the ITCZ behaves at higher latitudes where these assumptions break down.

We examine potential controls on the ITCZ's movement using a moist Earth GCM of varying seasonal forcing and rotation rate. We evaluate previous predictors for the ITCZ-namely, the maximum low-level MSE, Hadley cell extent, and EFE—as well as angular momentum-conserving theories of the Hadley cell's poleward extent, in a wide parameter space of rotation rates and seasonal forcing. In addition, we assess the interaction of BL dynamics with the large-scale circulation. As a result, our work evaluates both thermodynamic and dynamic arguments for the ITCZ's position in perpetually solstitial and slowly rotating seasonal largescale circulations. In section 2 , we describe the model and experimental setup. In section 3, we focus on the unique eternal-solstice case at Earth's rotation rate and evaluate relevant CQE theories. In section 4, we analyze the general circulation of our rotation rate experiments. Then in section 5 , we present analysis of the BL dynamics and radiative energy balance for all experiments.

\section{Methods}

We use the moist idealized three-dimensional GCM described in Frierson et al. (2006) and Frierson (2007) 
based on the Geophysical Fluid Dynamics Laboratory (GFDL) spectral dynamical core, but with two major changes: 1) the addition of a seasonal cycle and 2) longwave optical depths that do not depend on latitude.

Radiative heating and cooling are represented by gray radiative transfer, in which radiative fluxes are only a function of temperature, thus eliminating water vapor feedback. There is no diurnal cycle. The convection parameterization is a simplified Betts-Miller scheme, described fully in Frierson (2007). The scheme relaxes the temperature and moisture profiles of convectively unstable columns to a moist adiabat with a specified relative humidity ( $70 \%$ in these simulations) over a fixed relaxation time $(2 \mathrm{~h})$. Standard drag laws are used to calculate surface fluxes, with drag coefficients determined by a simplified Monin-Obukhov scheme. The BL scheme is a standard $K$-profile scheme with diffusivities consistent with the simplified Monin-Obukhov theory. The lower boundary is a zonally symmetric slab mixed layer ocean with a constant depth of $10 \mathrm{~m}$ in the control case, corresponding to a heat capacity $C$ of $1 \times$ $10^{7} \mathrm{~J} \mathrm{~m}^{-2} \mathrm{~K}^{-1}$ and a thermal inertia time scale $\tau_{f} \sim$ 20 days, where $\tau_{f}=C / 4 \sigma \bar{T}^{3}$ and $\bar{T}=285 \mathrm{~K}$ (Mitchell et al. 2014). Thus, sea surface temperatures are prognostic and adjust to ensure the surface energy budget is closed in the time mean.

We implement the seasonally varying TOA insolation prescription from Hartmann (1994, 347-349), wherein the declination angle is approximated by a Fourier series that provides an empirical fit to Earth's current insolation. The prescription accounts for Earth's eccentricity.

The latitude-dependent optical depth of the original model was suited for an equinoctial framework and is thus inappropriate for our seasonal framework where bands of precipitation and humidity fluctuate in latitude over the course of a year. In our model, the prescribed optical depth is independent of latitude, becoming a function only of pressure with a linear component, describing the effect of well-mixed greenhouse gases, and a quartic component, capturing the effect of water vapor confined to the lower troposphere. Parameters of the pressure-dependent optical depth are as in Frierson et al. (2006), except the surface value of optical depth $\tau_{0}=(1 / 2)\left(\tau_{0 e}+\tau_{0 p}\right)$, where $\tau_{0 e}$ and $\tau_{0 p}$ represent the equator and pole, respectively.

The model uses the primitive equations with T42 spectral resolution and 25 unevenly spaced vertical levels, with greater resolution in the BL. We conduct two primary sets of simulations: 1) we reduce the frequency of the seasonal cycle down to the extreme case where the planet is in eternal solstice, with all other parameters kept Earth-like [default parameters as in Frierson et al. (2006)], to observe ITCZ behavior over the longer time scales of fixed insolation, and 2) we adjust the rotation rate of an Earth-like planet from 4 times larger than Earth's value down to 32 times smaller than Earth's value, with all parameters other than rotation rate (insolation, radius, gravity, etc.) kept Earth-like and the insolation cycle kept fixed.

All simulations are run for $10 \mathrm{yr}$. Values for the eternal-solstice simulation are averaged over the final year ( $9 \mathrm{yr}$ of spinup). Values for the seasonal cases are composited over the last $8 \mathrm{yr}$ ( $2 \mathrm{yr}$ of spinup). All solstitial time averages for each year of the seasonal cases are taken during a 40-day period centered around the time of maximum zonally averaged Northern Hemisphere precipitation.

\section{Evaluating thermodynamic mechanisms via the eternal-solstice case}

A thermodynamic theory for the location of the convergence zone is based on the assumptions of a moist adiabatic vertical thermodynamic profile in statistical equilibrium (i.e., CQE) and an angular momentumconserving meridional circulation that constrains freetropospheric temperature gradients to be weak. CQE thus connects upper-tropospheric temperature to lowlevel MSE and, when coupled with dynamical circulation theory, argues that the maximum zonal-mean precipitation should occur just equatorward of the latitude of maximum zonal-mean subcloud MSE (Emanuel et al. 1994; Privé and Plumb 2007). The low-level, zonalmean MSE $[m]=c_{p}[T]+[\Phi]+L_{v}[q]$, where the square brackets indicate zonal mean, is strongly correlated with surface temperature over a saturated surface, as in our aquaplanet simulations. Examining the ITCZ in the eternal-solstice experiment provides further insight into the CQE argument by effectively eliminating energetic adjustment time scales that might otherwise conflate seasonal effects.

The top-left panel of Fig. 1 shows the zonal-mean precipitation for the eternal-solstice case at Earth's rotation rate. Maximum zonal- and time-mean MSE in this case is located at the summer pole (Fig. 2, top panel; vertical green line). The ITCZ, though, remains at low latitudes. Indeed, the maximum zonal- and time-mean precipitation occurs at approximately the same latitude for both the seasonal and eternal-solstice case, around $20^{\circ}-25^{\circ} \mathrm{N}$. This is seemingly at odds with CQE arguments. However, the thermal stratification set by moist convection assumed by CQE can only be communicated effectively by gravity waves in the tropics, where WTG holds. At the pole in the eternal-solstice case, WTG is 

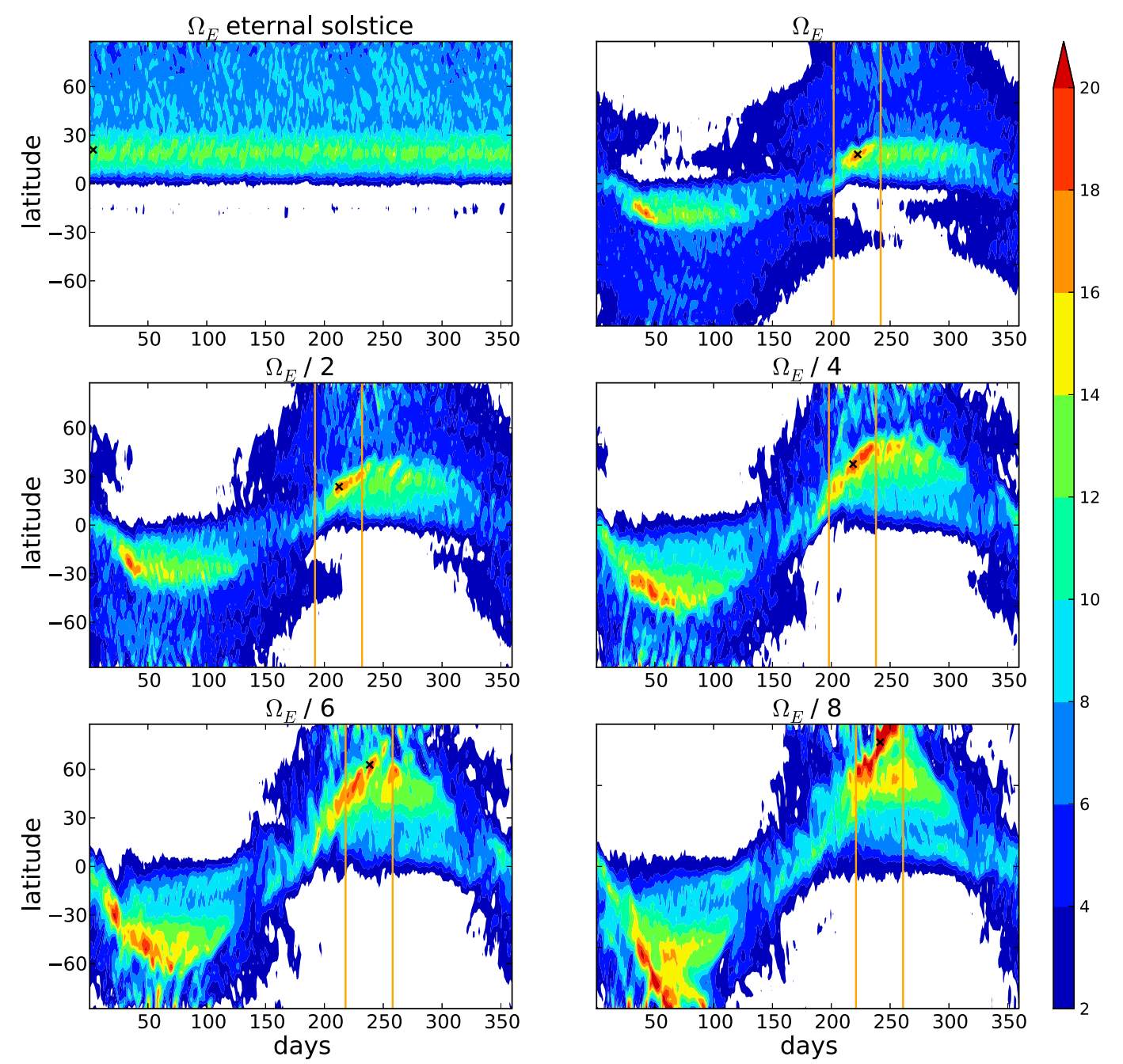

FIG. 1. Zonal-mean precipitation $\left(\mathrm{mm} \mathrm{day}^{-1}\right)$ for all cases: the eternal-solstice simulation and five seasonal cases of varying rotation rate. Orange vertical lines in the seasonal cases indicate time over which solstitial time averages are taken and represent the 40-day period between 20 days before the time of maximum zonal-mean Northern Hemisphere precipitation (black cross) and 20 days after that time.

not a good approximation and hence in this case, lowlevel MSE and the ITCZ can be greatly separated.

Alternatively, the latitudinal separation between a polar MSE maximum and a subtropical ITCZ may be understood through the theory of Emanuel (1995), which, extending the arguments of Plumb and Hou (1992) to a moist convective atmosphere, states that a cross-equatorial Hadley circulation needs to exist (in place of a radiative equilibrium response) when the curvature of the subcloud moist entropy exceeds a critical value. To test the criticality condition, we run a radiative-convective eternal-solstice simulation at Earth's rotation rate and calculate the critical curvature as in (10) of Emanuel (1995). In the top panel of Fig. 2, we show both sides of this equation: the subcloud moist entropy curvature of the radiative-convective simulation (solid line) and the critical curvature (dotted line) for Earth's rotation rate. Figure 2 clearly shows that a circulation is necessary equatorward of their intersection at $\sim 20^{\circ}$.

Thus, importantly, the critical condition is consistent with the existence of a low-latitude circulation in our (dynamically equilibrated) eternal-solstice case, despite zonal-mean MSE maximizing at the pole (Fig. 2, top panel; vertical green line). The ITCZ of the dynamically equilibrated eternal-solstice simulation (Fig. 2, top panel; vertical blue line) occurs just equatorward of the intersection given by the radiative-convective critical condition, consistent with the above arguments. Additionally, the subcloud moist entropy curvature of the dynamically equilibrated state (Fig. 2, top panel; dashed line) largely overlaps that of the radiative-convective 

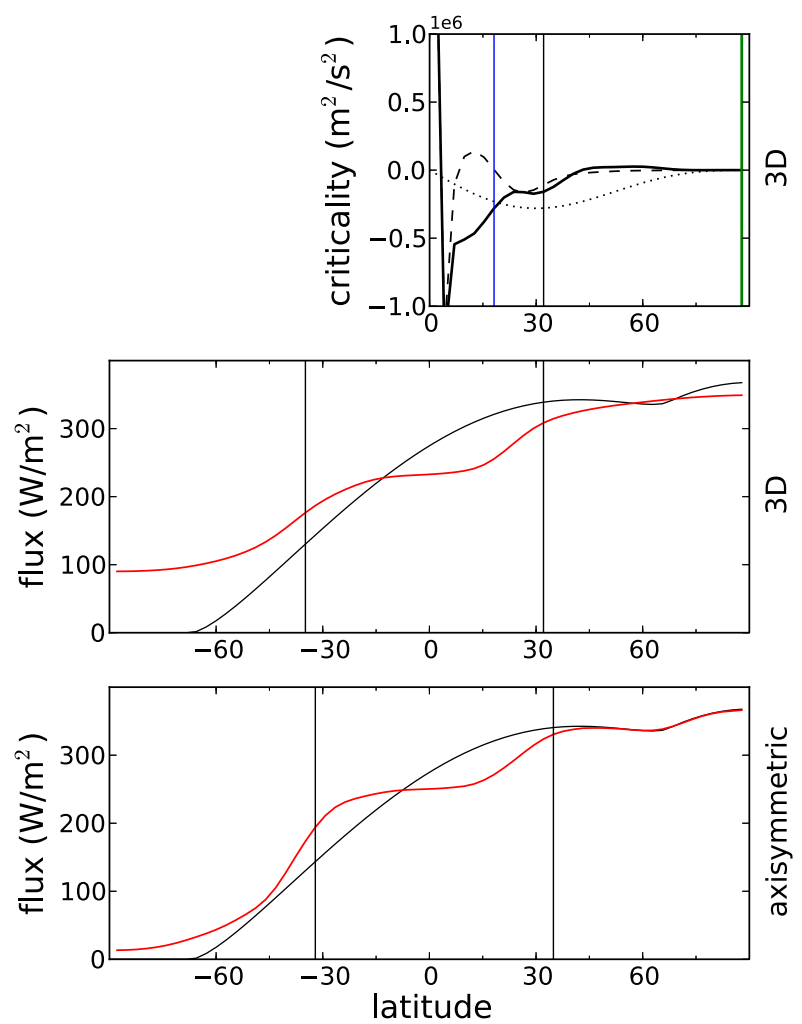

FIG. 2. (top) Critical condition as from Emanuel (1995). Solid line gives the curvature calculated from the subcloud moist entropy distribution of the radiative-convective eternal-solstice experiment according to $\partial / \partial \phi\left\{\left(\cos ^{3} \phi / \sin \phi\right)\left(T_{s}-T_{t}\right) \partial s_{b} / \partial \phi\right\}$, where $s_{b}$ is the moist entropy taken at $\sigma=0.85$ and $T_{t}$ is the temperature at $\sigma=0.20$. Dashed line gives the curvature calculated from the equilibrated subcloud moist entropy distribution of the eternalsolstice simulation at Earth's rotation rate. The dotted line gives the critical curvature, $-4 \Omega^{2} a^{2} \cos ^{3} \phi \sin \phi$. Vertical blue line and green line represent latitude of maximum $\overline{[P]}$ and latitude of maximum $\overline{[\mathrm{MSE}]}$ in the equilibrated eternal-solstice simulation, respectively. Vertical black lines indicate northern and southern extents of winter Hadley cell of the equilibrated eternal-solstice simulation, where Hadley cell extent is defined as the latitude where the streamfunction, taken at the pressure level of its maximum value, reaches $2 \%$ of that maximum value. Insolation (black) and zonal- and time-mean OLR (red) for (middle) the equilibrated eternal-solstice simulation and (bottom) an axisymmetric eternalsolstice simulation, both at Earth's rotation rate. Vertical black lines are Hadley cell extents as defined in (top).

case in the subcritical extratropics, while the latitude at which it meets the critical condition is $\sim 10^{\circ}$ equatorward of that for the radiative-convective case. To demonstrate the similarities between these arguments and the angular momentum-conserving arguments of Held and Hou (1980), we include the TOA energy balance for the eternal-solstice case in the middle and bottom panels of Fig. 2, which we will return to in section 5a.

For varying rotation rates, regions where entropy gradients are supercritical extend farther poleward as rotation rate decreases; as shown in section 4 , the corresponding Hadley circulations however expand to an even greater degree. Using critical curvatures calculated from the moist entropy distributions of the simulations themselves yields slightly better predictions of the circulation extent, but the results are complicated by two factors: 1) the circulation reconfigures the subcloud moist entropy distribution significantly from the conditions assumed in deriving the criticality, and 2) the criticality condition predicts the presence or absence of a circulation and thus is a predictor of the Hadley cell extent, rather than the ITCZ. Hence, this criticality argument cannot be easily translated into either a predictor or a quantitative diagnostic of the ITCZ position for our simulations. Despite these limitations, further exploration of the critical curvature condition as applied to the ITCZ of varying climates seems a worthwhile avenue for future work.

The eternal-solstice simulation at Earth's rotation rate also addresses the impact of heat capacity, which has been shown to affect the ITCZ's movement (Fein and Stephens 1987; Xie 2004; Pierrehumbert 2005; Bordoni and Schneider 2008; Abbot and Pierrehumbert 2010; Donohoe et al. 2014). Maximum insolation in the eternal-solstice case is fixed at the pole for $10 \mathrm{yr}$, much longer than the time scale for atmospheric adjustments, and still the precipitation maximum lies at low latitudes. Thus, it appears that reducing the time scale for atmospheric adjustments even further would not have any impact on the ITCZ migration. Indeed, we run simulations of varying surface heat capacities and find that there is little change in the position of the ITCZ as the heat capacity is decreased beyond the control (not shown). In addition, a lack of baroclinic activity in the extratropics of the eternal-solstice case suggests baroclinic instability, through influences on the summer Hadley cell, plays no role in limiting the ITCZ's poleward migration during solstice in our simulations (confirmed by axisymmetric runs).

The subtropical ITCZ in the eternal-solstice case, when combined with results that atmospheric and lowerboundary thermal inertia cannot push an ITCZ to the pole, suggests that planetary parameters must be important for ITCZ migrations in other planetary settings such as Mars and Titan. We therefore explore the influence of decreasing rotation rate in the next section.

\section{Rotation rate experiments}

Here we run Earth-like simulations for varying rotation rates to explore the conditions under which the ITCZ can seasonally migrate poleward. This is roughly equivalent to varying radius since both parameters 

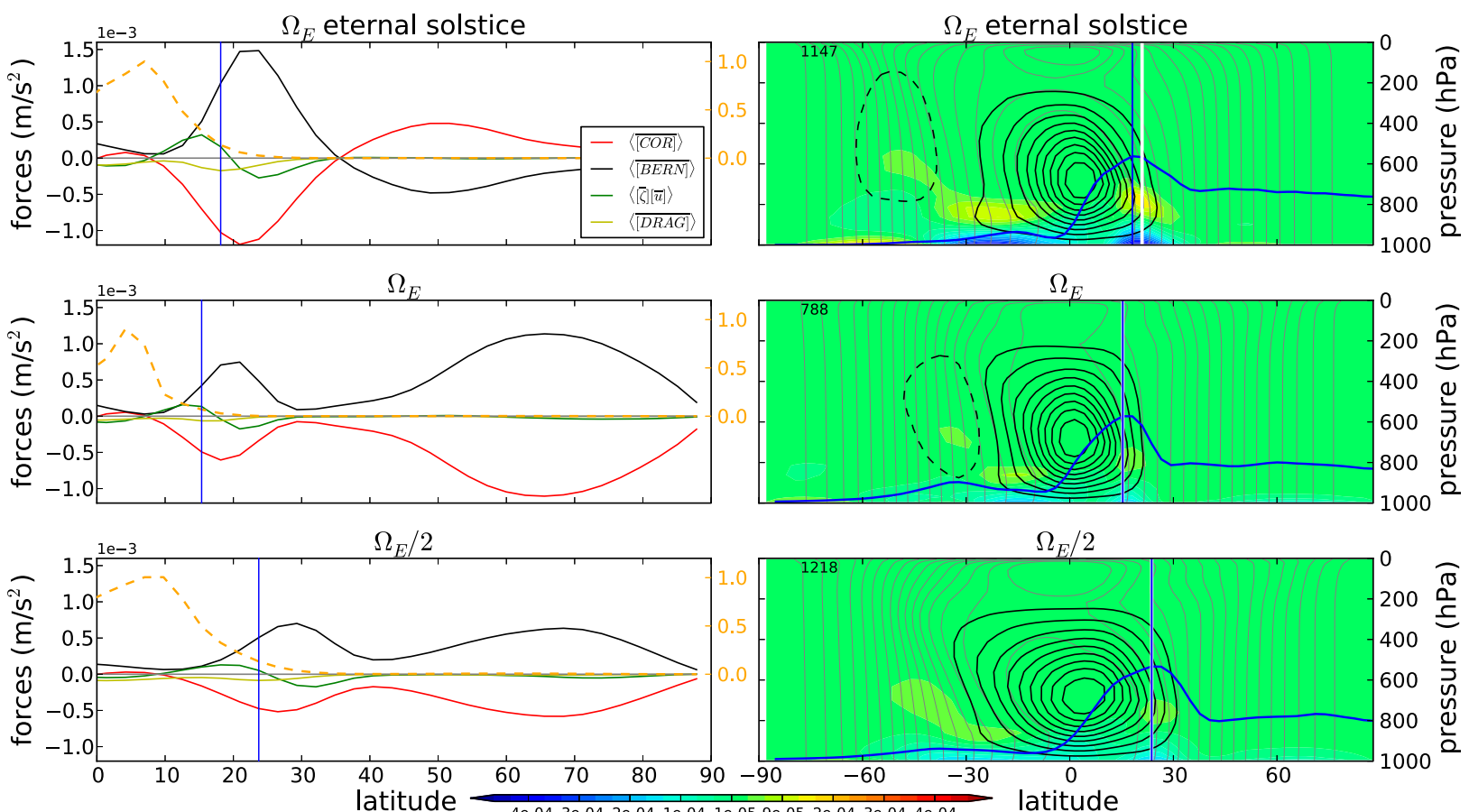

FIG. 3. (left) Summer hemisphere forces on left $y$ axis, with positive values denoting a northward force and where BERN, COR, and DRAG correspond to the Bernoulli gradient, Coriolis, and frictional forces, respectively. Each force is diagnosed directly from simulation. Values of $G$, from Schneider and Bordoni (2008), are on right $y$ axis. Vertical blue line represents the latitude of maximum $\overline{[P]}$. Vertical integration taken from the surface to $\sigma=0.85$. Solstitial time averages taken over period shown in Fig. 1. (right) Zonally and solstitially averaged streamfunction, angular momentum, drag, and precipitation. Streamfunction contours $\left(10^{9} \mathrm{~kg} \mathrm{~s}^{-1}\right.$; black; solid lines counterclockwise) are $10 \%$ of maximum, printed in top-left corner. Gray contours are angular momentum contours $\left(\Omega a^{2} / 17\right.$ interval). Color contours are drag force $\left(\mathrm{m} \mathrm{s}^{-2}\right)$. Precipitation $\overline{[P]}$ in blue (units not shown). Solstitial time averages taken over period shown in Fig. 1. Vertical white line represents latitude of maximum vertically integrated BL drag force. Vertical blue line represents latitude of maximum $\overline{[P]}$.

similarly impact Hadley cell width (Held and Hou 1980). From Fig. 1, the ITCZ moves farther off the equator toward the summer pole with each decrease in rotation rate, consistent with the expanding annual-mean Hadley cell for slower rotation rates found in previous studies (Williams 1988; Navarra and Boccaletti 2002; Walker and Schneider 2006; Mitchell and Vallis 2010; Mitchell et al. 2014; Dias Pinto and Mitchell 2014; Kaspi and Showman 2015). We run simulations with rotation rates down to 32 times smaller than Earth's, but they are not shown because their circulations are similar in structure to that of the $\Omega / \Omega_{E}=1 / 8$ case. Similarly, the $\Omega / \Omega_{E}=2$ and $\Omega / \Omega_{E}=4$ cases are not shown owing to their circulation structures largely resembling that of the control case, though their ITCZs are indeed closer to the equator than the control (see Fig. 5).

For $\Omega / \Omega_{E} \leq 1 / 8$, the planet enters a regime where the winter Hadley cell becomes global, precluding any significantly farther migration with larger decreases in rotation. Note that local maxima in MSE exist at the pole during solstice in all cases (not shown), consistent with the small "patches" of polar precipitation separated from the main ITCZ band in the quickly rotating cases. Indeed, for $\Omega / \Omega_{E} \leq 1 / 8$, a transient maximum in zonalmean precipitation exists at the summer pole (Fig. 1). This polar maximum is however separate from the ITCZ, which is the precipitation maximum at lower latitudes associated with the region of strongest convergence in the ascending branch of the Hadley cell $\left(\sim 60^{\circ} \mathrm{N}\right.$ in the $\Omega / \Omega_{E}=1 / 8$ case $)$.

The right columns of Figs. 3 and 4 show the zonally averaged meridional circulation and angular momentum contours during solstice for all rotation cases. Consistent with the precipitation, the cross-equatorial Hadley cell expands farther poleward for each decrease in rotation rate. Additionally, the summer cell is negligible when compared to the cross-equatorial winter cell for all simulations during the averaged solstitial time period. The right columns of Figs. 3 and 4 also show zonal-mean precipitation averaged over the solstitial time period, demonstrating the association between the ITCZ and the Hadley cell structure. In aquaplanet simulations of Earth with negligible surface thermal inertia, as the seasonal cycle transitions from equinox to 

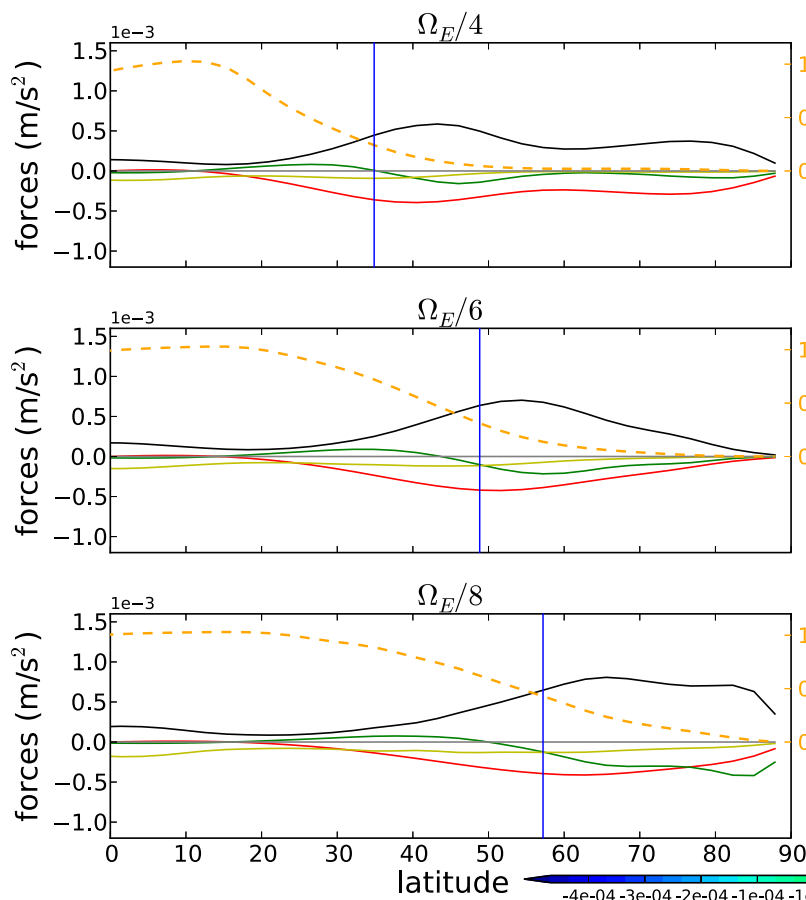

FIG. 4. As in Fig. 3, but for more slowly rotating cases.
$\Omega_{E} / 4$

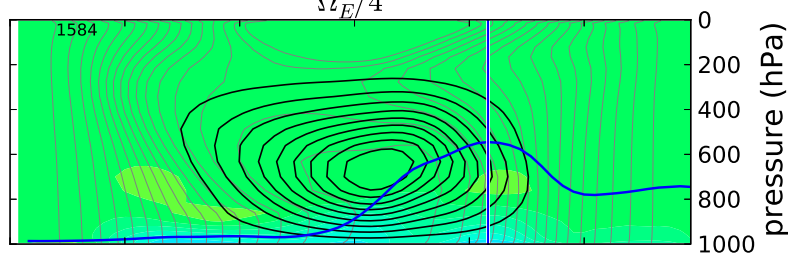

$\Omega_{E} / 6$

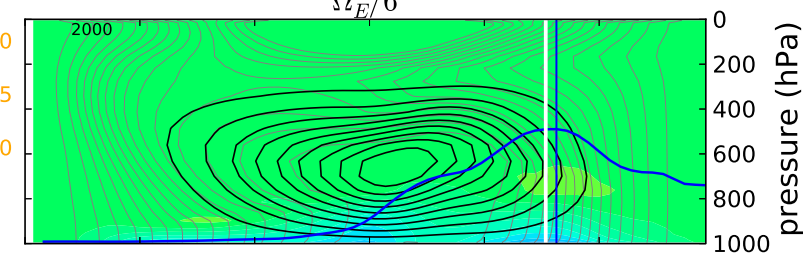

$\Omega_{E} / 8$

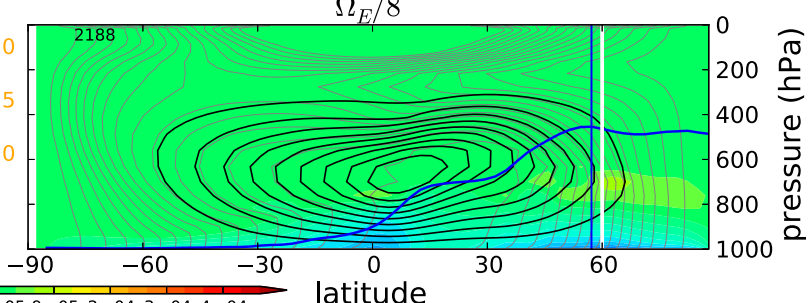

solstice-representing monsoon onset over landdominated regions such as the Asian monsoon regionthe winter Hadley cell strengthens and its ascending upper branch becomes more angular momentum conserving (middle row in Fig. 3) (Lindzen and Hou 1988; Bordoni and Schneider 2008; Schneider and Bordoni 2008). At solstice, upper-level easterlies shield the circulation from energy-containing midlatitude eddies and the eddy momentum flux divergence is weak, thus allowing streamlines to follow angular momentum contours in most of the upper branch of the crossequatorial winter cell. We observe similar solstitial dynamics as the rotation rate is decreased in our simulations: streamlines in the ascending region of all cases generally follow angular momentum contours, and in all cases a region of upper-level easterlies (not shown) is maintained by the winter circulation, which continues to expand latitudinally with decreasing rotation rate down to $\Omega / \Omega_{E}=1 / 8$, where the circulation finally becomes global.

Thus, a regime change occurs after $\Omega / \Omega_{E}=1 / 8$, wherein three conditions hold for planets with that rotation rate and slower: 1) the winter cross-equatorial cell extends from pole to pole and has a latitudinally wide region of updraft as opposed to the meridionally narrow ascent region seen in the more quickly rotating cases, 2) eddy momentum flux divergence is weak at all latitudes, and 3) the ITCZ does not move past $\sim 60^{\circ}-70^{\circ}$
(Fig. 4, bottom-right panel; vertical blue line) despite the circulation becoming global, though a shorter-lived precipitation maximum is observed at the summer pole. A similar regime change was noted in the nonseasonal experiments of Walker and Schneider (2006), wherein the upper branches of the circulations for slowly rotating planets were nearly angular momentum conserving around the latitude of the Hadley streamfunction extremum.

\section{Predictors for the ITCZ}

Here we evaluate relevant predictors for the ITCZnamely, the Hadley cell extent, the maximum low-level MSE, and the EFE. As in the previous section, the zonally averaged low-level MSE $\overline{[m]}$ is taken at $850 \mathrm{hPa}$, with the overbar denoting solstitial average. The Hadley cell extent $\phi_{H}$ is defined as the latitude where the crossequatorial streamfunction, taken at the pressure level of its maximum value, reaches $5 \%$ of that maximum value in the summer hemisphere (Walker and Schneider 2006). Finally, the EFE defined in Kang et al. (2008) as where the energy flux $F=\langle\overline{[m v]}\rangle$ reaches zero, with the angle brackets denoting vertical integration. However, since $F$ does not vanish until the pole for $\Omega / \Omega_{E}=1 / 2$ and slower during solstice, here we define the EFE as the latitude where the flux $F$ reaches $5 \%$ of its maximum value, similar to the Hadley cell extent definition. Figure 5 shows these predictors, each normalized to the 


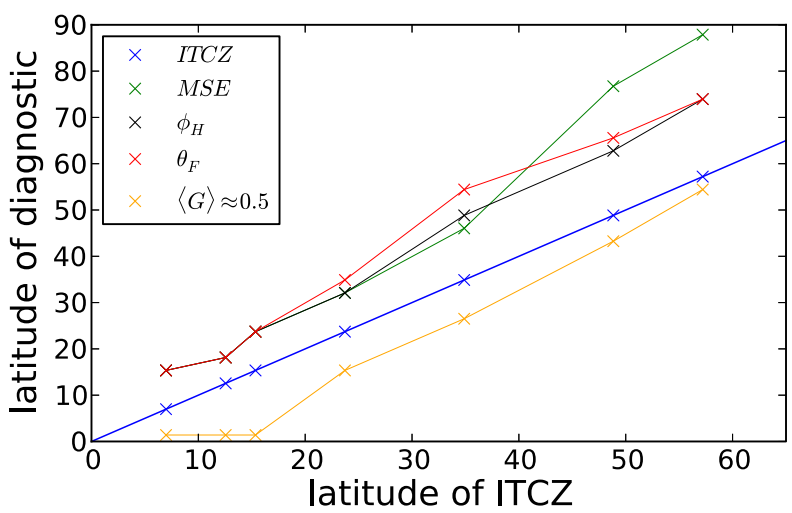

FIG. 5. Zonal- and time-mean maximum low-level MSE (green), Hadley cell extent (black), EFE (red), and latitude where $\langle G\rangle \approx 0.5$ (orange) as compared to the ITCZ (blue) for different rotation cases. Blue line is $1: 1$. From left to right, rotation rates $\Omega / \Omega_{E}$ are $4,2,1,1 / 2,1 / 4,1 / 6,1 / 8$.

maximum zonally and solstitially averaged precipitation (the ITCZ), for all rotation cases. It also shows the latitude where the function $G=0.5$, with $G$ defined in Schneider and Bordoni (2008). We return to this predictor in section $5 b$.

The MSE maximum, Hadley cell extent, and EFE occur poleward of the latitude of the ITCZ for all cases. These three values are generally well correlated (particularly at faster rotation rates). Indeed, the Hadley cell extent and EFE, physically related to one another (and, by definition, nearly equivalent), are approximately collocated for each rotation rate. It is also clear from Fig. 5 that the ITCZ is associated with the ascending branch of the cross-equatorial winter Hadley cell but generally lies equatorward of its edge. However, the distance of separation between the ITCZ and the winter Hadley cell extent appears to increase with decreasing rotation rate. Once the circulation enters the slowly rotating regime, the ITCZ remains approximately at $\sim 60^{\circ}$ while the Hadley cell extent remains at $\sim 75^{\circ}-80^{\circ}$. We also note the larger discrepancy between the latitude of maximum MSE and the Hadley cell extent in the $\Omega / \Omega_{E}=1 / 6$ and $\Omega / \Omega_{E}=1 / 8$ cases. This is consistent with the transient polar convection being separated from the large-scale circulation in these cases-a feature also observed in GCM models of Titan's atmosphere (Mitchell and Lora 2016).

That the maximum precipitation is slightly equatorward of the maximum MSE during solstice on Earth has been noted in theory, models, and observations (Privé and Plumb 2007; Bordoni and Schneider 2008). Small discrepancies between the EFE and the ITCZ in Earth settings have also been observed in idealized models and reanalysis data (Kang et al. 2008; Bischoff and Schneider 2014, 2016; Adam et al. 2016), emphasizing the need for a comprehensive theory of the ITCZ position that couples considerations of both the energy balance and the angular momentum balance, particularly in strongly off-equatorial regimes. In the next section, we work toward developing an understanding of the position of the ITCZ based on BL dynamics and TOA energy balance.

\section{Evaluating dynamical mechanisms behind the ITCZ position}

The flow that leads to the ITCZ is part of the lower branch of the cross-equatorial winter Hadley cell. Using the case of northern summer solstice as an example, this flow is the northward BL flow between the equator and the northern edge of the winter cell in the summer hemisphere. The edge of the winter cell itself is where the flow vanishes, at a latitude analogous to the EFE or the maximum MSE, and can be determined by TOA energy balance (Held and Hou 1980; Lindzen and Hou 1988; Satoh 1994; Caballero et al. 2008). Meridional forces in the $\mathrm{BL}$, primarily the northward pressure gradient force and southward Coriolis force, govern the zonal-mean flow. However, while eddy momentum flux divergence and vertical advection are very small in the BL, nonlinear advective forces are not and so the flow is not completely in geostrophic balance. The pressure gradient, Coriolis, and nonlinear forces combine into a northward force that balances the southward drag. Since the flow must vanish farther poleward at the Hadley cell edge-and indeed all the forces must vanish there, except for in the slowly rotating regime where they cannot vanish before reaching the pole-there is a flow transition that must occur that leads to the ITCZ. Our simulations demonstrate this mechanism and also provide a scaling for the latitude of the ITCZ's poleward excursion with rotation rate.

\section{a. TOA energy balance}

The mechanism behind the ITCZ, as it appears from our simulations, is intimately tied to previous work done by Held and Hou (1980) and in particular by Caballero et al. (2008), who applied the energy balance arguments of Held and Hou (1980) to the solstitial circulation in order to determine the extent of the cross-equatorial winter Hadley cell into the summer hemisphere. The simulated cross-equatorial circulations for all rotation rates approach the angular momentum-conserving solution, in which the potential temperature in the free troposphere adjusts through thermal wind balance to the angular momentum-conserving winds. Together with energy conservation constraints (i.e., the energy 


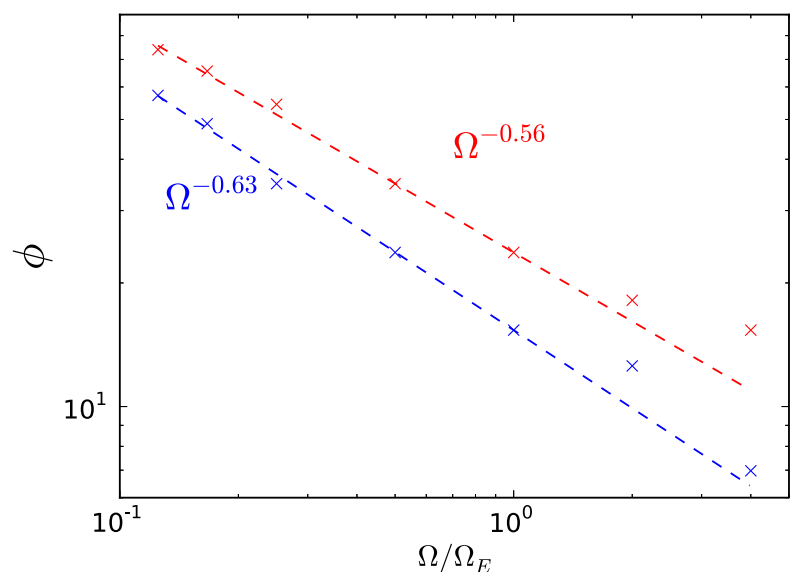

FIG. 6. Rotation rate vs latitudes of ITCZ (blue) and EFE (red). Dashed lines represent scaling fits to cases with rotation rate $\Omega / \Omega_{E}=1 / 8$ and greater (including $\Omega / \Omega_{E}=2$ and $\Omega / \Omega_{E}=4$ ) for both values (color corresponds to color of crosses).

budget is closed within the Hadley cell and there is temperature continuity with the radiative-convective equilibrium profile at the cell's poleward boundary), these angular momentum-conserving arguments can be used to determine the latitude of the ascending branch of the winter Hadley cell, corresponding to where the energy flux and boundary layer forces vanish just poleward of the ITCZ position, as described above.

Held and Hou (1980) find the Hadley cell extent to be dependent on the thermal Rossby number such that $\phi_{H} \propto(\mathrm{Ro})^{1 / 2}$ for Ro $=g H \Delta_{H} / \Omega^{2} a^{2}$, where $H$ is the tropopause height and $\Delta_{H}$ is the pole-to-equator radiative-convective equilibrium temperature difference. Caballero et al. (2008) applied similar arguments to seasonal insolation and found that the solstitial Hadley cell extent followed a different power law: $\phi_{H} \propto(\mathrm{Ro})^{1 / 3}$. Figure 6 shows the scalings of the ITCZ and the EFE with rotation rate. Cases slower than $\Omega / \Omega_{E}=1 / 8$ were not included in the scaling because the ITCZ's progression with rotation rate is limited by the pole beyond that point; that is, once the Hadley cell is global, it remains so for slower rotation rates.

Since Ro scales as $\Omega^{-2}$, the solstitial Hadley extent as determined by Caballero et al. (2008) scales as $\Omega^{-2 / 3}$, and Fig. 6 shows a similar scaling in our simulationsboth for the energy flux equator and the latitude of the ITCZ itself-despite the fact that Caballero et al. (2008) used dry axisymmetric theory. This may be associated with the dynamic characteristics of solstice in an aquaplanet simulation: during solstice, the upper branch of the winter Hadley cell is approximately angular momentum-conserving at the latitude of ascent, with local Rossby numbers $\mathrm{Ro} \geq 0.6$, signifying the relatively weak impact of eddy momentum flux divergence (Bordoni and Schneider 2008; Schneider and Bordoni 2008), thereby approximating axisymmetric conditions. As a caveat to the similarities just described, we should note that the power laws of Held and Hou (1980) and Caballero et al. (2008) employ the small-angle approximation, which is most likely inappropriate for our most slowly rotating cases.

TOA energy balance further illuminates the lowlatitude ascent in the eternal-solstice case despite temperatures maximizing in that simulation at the pole. Aforementioned energy conservation constraints argue for the Hadley cell edge to coincide with the intersection of the insolation profile and zonal-mean outgoing longwave radiation (OLR). The zonal-mean OLR, SST, and low-level MSE all exhibit approximately the same meridional profile in our eternal-solstice simulation, maximizing at the pole, but the OLR intersects with the insolation at a latitude just less than $\sim 60^{\circ}$ (see Fig. 2, middle panel). Note from the bottom panel of Fig. 2 that we additionally run an axisymmetric eternal-solstice case, which gives a slightly better estimate of the winter Hadley cell extent using TOA energy balance arguments given the absence of baroclinic eddies that diverge energy out of the subtropics.

The above arguments, however, apply specifically to the Hadley cell extent rather than the ITCZ. The latitudinal separation between the ITCZ and predictors related to the Hadley cell, which can be quite large in some of our rotation rate experiments, suggests the importance of the BL momentum budget, since precipitation is collocated with lower-level convergence and $\mathrm{BL}$ forces drive lower-level flow.

\section{b. Boundary layer dynamics}

Following Schneider and Bordoni (2008), the BL steady-state momentum equations with drag represented as Rayleigh drag with damping coefficient $\varepsilon$ are

$$
\begin{aligned}
-(f+\bar{\zeta}) \bar{v} & \approx-\varepsilon \bar{u} \quad \text { and } \\
(f+\bar{\zeta}) \bar{u}+\frac{\partial \bar{B}}{\partial y} & \approx-\varepsilon \bar{v},
\end{aligned}
$$

where $\bar{B}=\bar{\Phi}+\left(\bar{u}^{2}+\bar{v}^{2}\right) / 2$ is the mean barotropic Bernoulli function with geopotential $\Phi$ and the overbar again denotes the time mean. Formulating the momentum equation this way means that the Bernoulli gradient accounts for the pressure gradient force as well as nonlinear contributions unaccounted for by the $\bar{\zeta} \bar{u}$ term. Aside from approximating turbulent drag as Rayleigh drag, the other assumptions made here are that contributions from eddy momentum flux divergence and 
vertical advection are negligible, and indeed in our simulations those terms are about an order of magnitude smaller than the retained Coriolis, Bernoulli gradient, drag, and $\bar{\zeta} \bar{u}$ terms (we include the Rayleigh drag approximation here because it is used in the calculation of $G$ later in this section, but the drag force plotted in Figs. 3 and 4 is diagnosed directly from the model, calculated by horizontal diffusion).

Figures 3 and 4 give a summary of these forces for each rotation case during northern summer solstice and the eternal-solstice case, with all values vertically integrated over the $\mathrm{BL}$. We define the $\mathrm{BL}$ as ending at $850 \mathrm{hPa}$, though note that for the more slowly rotating cases, the lower branch of the circulation reaches the free troposphere. For all cases, the ITCZ is well correlated with the latitude of maximum BL drag, just equatorward of the edge of the winter Hadley cell. Indeed, in the control case the ITCZ and latitude of maximum drag are exactly collocated.

As rotation slows and the convergence zone widens, the convergence zone force balance changes from primarily geostrophic balance to something closer to cyclostrophic balance, with the nonlinear $\bar{\zeta} \bar{u}$ force becoming a dominant contributor to balancing the Bernoulli gradient force. Also in the slowly rotating cases, regions of strong meridional drag exist at equatorial latitudes. Corresponding secondary maxima of precipitation (see Figs. 3 and 4) and vertical velocity (not shown) occur in these regions, but they are located $\sim 10^{\circ}-15^{\circ}$ downstream of the maximum drag. Pauluis (2004) describes the dynamics of a similar secondary precipitation maximum near the equator in axisymmetric cross-equatorial Hadley circulations. The downstream displacement of this secondary precipitation maximum in our simulations $\left(\sim 10^{\circ}-15^{\circ} \mathrm{N}\right)$ relative to that seen in the axisymmetric simulations of Pauluis (2004) might be indicative of the increasing role of nonlinear terms in the BL momentum budget as rotation rate is decreased.

The left columns of Figs. 3 and 4 show that the same approximate force balance holds for each case. Convergence occurs as the flow transitions from a BL balance primarily between the Bernoulli gradient force and friction to a BL balance primarily between the Bernoulli gradient force and the Coriolis force, a transition zone previously defined and described in Schneider and Bordoni (2008) via the nondimensional function $G=\varepsilon^{2} /\left\{\varepsilon^{2}+(f+\overline{[\zeta]})^{2}\right\}$ (Figs. 3 and 4, left columns; dashed orange lines). We calculate $G$ using $\varepsilon=C_{d}\left(\overline{[u]^{2}}+\overline{[v]^{2}}\right)^{1 / 2} / H_{0}$, with $C_{d}$ being the surface drag coefficient of momentum and $H_{0}$ being the height of the BL (Lindzen and Nigam 1987). Schneider and Bordoni (2008) approximate the ITCZ as the region where $G \approx 0.5$, a descriptor that is not collocated but still correlated with the ITCZ in our simulations (see Fig. 5). The transition zone occurs over a larger latitudinal range as rotation rate decreases, consistent with the widening winter Hadley cell and more extensive regions of convergence and precipitation in those cases. Where the Bernoulli gradient force and friction are balanced equatorward of the ITCZ, the zonal- and time-mean absolute vorticity $\overline{[\eta]}$ is approximately zero in every case (not shown). This is consistent with the work of Tomas and Webster (1997), in which the $\overline{[\eta]}=0$ latitude demarcates between divergence and convergence, with divergence equatorward of that latitude and convergence associated with inertial instability-producing the ITCZ-poleward of that latitude.

It is also apparent from the left columns of Figs. 3 and 4 that the ITCZ is associated with the maximum northward Bernoulli gradient force, consistent with the ITCZ being well correlated with the maximum MSE gradient in all cases (not shown) given that temperature gradients determine pressure gradients, which have been argued to drive the convergence-zone low-level flow (Lindzen and Nigam 1987; Tomas and Webster 1997; Pauluis 2004; Back and Bretherton 2009). However, because of the increasing relevance of nonlinear advective forces (i.e., the $\bar{\zeta} \bar{u}$ force) as the ITCZ moves farther from the equator in the more slowly rotating cases, the linear theories of Lindzen and Nigam (1987) and Back and Bretherton (2009) cannot be applied here, as they predict an ITCZ too far poleward in all but our control simulation. Indeed, the significance of the $\bar{\zeta} \bar{u}$ force implies that classic Ekman theory arguments describing flow-wherein the balance of forces is primarily between the pressure gradient force, the Coriolis force, and turbulent drag-are inappropriate when considering solstitial convergence-zone dynamics. According to Ekman theory, uplift maximizes where geostrophic vorticity maximizes, but in every one of our simulations geostrophic vorticity maximizes well poleward of the ITCZ during solstice (not shown), underlining the unsuitability of Ekman theory in solstitial situations and the importance of nonlinearity in determining the BL maximum convergence-a relationship also noted by Tomas et al. (1999).

\section{Summary and conclusions}

We conduct a suite of experiments to test various controls on the seasonal excursions of the ITCZ using an idealized, aquaplanet Earth GCM. We slow down the rotation rate of an otherwise Earth-like planet to examine the ITCZ as it migrates farther off the equator, and we additionally run an eternal-solstice experiment. 
Our noteworthy conclusions from these experiments are as follows:

- Classic ITCZ predictors-namely, maximum MSE, Hadley cell extent, and EFE-are generally collocated with each other but robustly lie poleward of the latitude of the ITCZ in our simulations, with the distance of separation increasing as the ITCZ moves farther from the equator. TOA energy balance, based on the axisymmetric theory of Held and Hou (1980), determines the latitude of these predictors (i.e., the latitude poleward of the ITCZ where the flow vanishes).

- The ITCZ is closely associated with maximum BL drag and Bernoulli gradient. Other precipitation maxima are correlated with regions of significant BL drag and Bernoulli gradient as well, including the transient precipitation maximum that appears at the summer pole for rotation rates $\Omega / \Omega_{E} \leq 1 / 8$. This maximum, however, is distinct from the ITCZ associated with the ascending branch of the Hadley circulation.

- The scalings of the ITCZ and EFE with rotation rate in our experiments are in good agreement with the dry axisymmetric $\Omega^{-2 / 3}$ scaling developed by Caballero et al. (2008).

- The eternal-solstice experiment demonstrates that the ITCZ is not always closely coupled with maximum zonal-mean MSE. This can occur because at Earth's rotation rate temperature gradients are constrained to be small only at lower latitudes and can instead be balanced through thermal wind at high latitudes. In this respect, while some convection can occur in the region of maximal $\mathrm{MSE}$ at the pole, the thermal stratification set by convection cannot be communicated elsewhere and cannot lead to a supercritical high-latitude MSE distribution.

Based on the ITCZ's behavior across a range of rotation rates and seasonal forcings, the conclusions above present a framework for understanding the location of the ITCZ based on energy balance and BL dynamics. Future work is necessary to fully expose the details of the underlying dynamics at play and develop a comprehensive theory for the ITCZ location.

Such relationships are important when thinking about analogous systems of seasonal convergence on other planets. Our results suggest that it is perhaps planetary parameters associated with the angular momentum budget (i.e., the rotation rate and/or radius) that are primarily responsible for Titan's and Mars's far-reaching convergence zones, rather than their low surface heat capacities, for instance. Indeed, the atmospheric circulations of the $\Omega / \Omega_{E}=1 / 8$ case and slower, which have intense polar precipitation and a global Hadley cell, are not too dissimilar from that of Titan, which itself has a rotation rate approximately one-sixteenth of Earth's and a radius onethird of Earth's. While we are not yet able to predict the poleward migration of the ITCZ given only planetary parameters, the axisymmetric scaling of Caballero et al. (2008) seems to be quite accurate when applied to our simulations, even though they are moist and eddy permitting. It is left for future work to further develop our diagnostic ITCZ understanding into a more prognostic theory that can be applied to any terrestrial planet with seasonally migrating convergence zones.

Acknowledgments. Simulations were carried out using the Hoffman2 Cluster hosted by UCLA's Institute for Digital Research and Education. The comments of our three anonymous reviewers and editor helped greatly focus and strengthen the final manuscript. S.B. acknowledges support by the National Science Foundation Grant AGS-1462544. S.F. and J.M. were supported by NASA Grants NNX12AI71G and NNX12AM81G, and S.F. was supported in part by UCLA's Eugene V. Cota-Robles Fellowship.

\section{REFERENCES}

Abbot, D. S., and R. T. Pierrehumbert, 2010: Mudball: Surface dust and Snowball Earth deglaciation. J. Geophys. Res., 115, D03104, doi:10.1029/2009JD012007.

Adam, O., T. Bischoff, and T. Schneider, 2016: Seasonal and interannual variations of the energy flux equator and ITCZ. Part I: Zonally averaged ITCZ position. J. Climate, 29, 3219-3230, doi:10.1175/JCLI-D-15-0512.1.

Arakawa, A., and W. H. Schubert, 1974: Interaction of a cumulus cloud ensemble with the large-scale environment, Part I J. Atmos. Sci., 31, 674-701, doi:10.1175/1520-0469(1974)031<0674: IOACCE $>2.0 . \mathrm{CO} ; 2$.

Back, L. E., and C. S. Bretherton, 2009: On the relationship between SST gradients, boundary layer winds, and convergence over the tropical oceans. J. Climate, 22, 4182-4196, doi:10.1175/2009JCLI2392.1.

Becker, E., G. Schmitz, and R. Geprägs, 1997: The feedback of midlatitude waves onto the Hadley cell in a simple general circulation model. Tellus, 49A, 182-199, doi:10.1034/ j.1600-0870.1997.t01-1-00003.x.

Bischoff, T., and T. Schneider, 2014: Energetic constraints on the position of the intertropical convergence zone. J. Climate, 27, 4937-4951, doi:10.1175/JCLI-D-13-00650.1.

_ and -2016 : The equatorial energy balance, ITCZ position, and double-ITCZ bifurcations. J. Climate, 29, 2997-3013, doi:10.1175/JCLI-D-15-0328.1.

Bordoni, S., and T. Schneider, 2008: Monsoons as eddy-mediated regime transitions of the tropical overturning circulation. Nat. Geosci., 1, 515-519, doi:10.1038/ngeo248.

Bouchez, A. H., and M. E. Brown, 2005: Statistics of Titan's south polar tropospheric clouds. Astrophys. J. Lett., 618, L53, doi:10.1086/427693. 
Broccoli, A. J., K. A. Dahl, and R. J. Stouffer, 2006: Response of the ITCZ to Northern Hemisphere cooling. Geophys. Res. Lett., 33, L01702, doi:10.1029/2005GL024546.

Caballero, R., R. T. Pierrehumbert, and J. L. Mitchell, 2008: Axisymmetric, nearly inviscid circulations in non-condensing radiative-convective atmospheres. Quart. J. Roy. Meteor. Soc., 134, 1269-1285, doi:10.1002/qj.271.

Chao, W. C., 2000: Multiple quasi equilibria of the ITCZ and the origin of monsoon onset. J. Atmos. Sci., 57, 641-652, doi:10.1175/1520-0469(2000)057<0641:MQEOTI>2.0.CO;2.

— , and B. Chen, 2001: Multiple quasi equilibria of the ITCZ and the origin of monsoon onset. Part II: Rotational ITCZ attractors. J. Atmos. Sci., 58, 2820-2831, doi:10.1175/ 1520-0469(2001)058<2820:MQEOTI >2.0.CO;2.

Chiang, J. C., and C. M. Bitz, 2005: Influence of high latitude ice cover on the marine Intertropical Convergence Zone. Climate Dyn., 25, 477-496, doi:10.1007/s00382-005-0040-5.

_- and A. R. Friedman, 2012: Extratropical cooling, interhemispheric thermal gradients, and tropical climate change. Annu. Rev. Earth Planet. Sci., 40, 383, doi:10.1146/ annurev-earth-042711-105545.

Dias Pinto, J. R., and J. L. Mitchell, 2014: Atmospheric superrotation in an idealized GCM: Parameter dependence of the eddy response. Icarus, 238, 93-109, doi:10.1016/j. icarus.2014.04.036.

Donohoe, A., J. Marshall, D. Ferreira, and D. Mcgee, 2013: The relationship between ITCZ location and cross-equatorial atmospheric heat transport: From the seasonal cycle to the Last Glacial Maximum. J. Climate, 26, 3597-3618, doi:10.1175/ JCLI-D-12-00467.1.

— D. M. Frierson, and D. S. Battisti, 2014: The effect of ocean mixed layer depth on climate in slab ocean aquaplanet experiments. Climate Dyn., 43, 1041-1055, doi:10.1007/ s00382-013-1843-4.

Emanuel, K. A., 1995: On thermally direct circulations in moist atmospheres. J. Atmos. Sci., 52, 1529-1534, doi:10.1175/ 1520-0469(1995)052<1529:OTDCIM>2.0.CO;2.

_ J. David Neelin, and C. S. Bretherton, 1994: On large-scale circulations in convecting atmospheres. Quart. J. Roy. Meteor. Soc., 120, 1111-1143, doi:10.1002/qj.49712051902.

Fein, J. S., and P. L. Stephens, Eds., 1987: Monsoons. Wiley, 632 pp.

Frierson, D. M., 2007: The dynamics of idealized convection schemes and their effect on the zonally averaged tropical circulation. J. Atmos. Sci., 64, 1959-1976, doi:10.1175/JAS3935.1.

, 2008: Midlatitude static stability in simple and comprehensive general circulation models. J. Atmos. Sci., 65, 1049-1062, doi:10.1175/2007JAS2373.1.

— shifts in slab ocean simulations of global warming. J. Climate, 25, 720-733, doi:10.1175/JCLI-D-11-00116.1.

— I. M. Held, and P. Zurita-Gotor, 2006: A gray-radiation aquaplanet moist GCM. Part I: Static stability and eddy scale. J. Atmos. Sci., 63, 2548-2566, doi:10.1175/JAS3753.1.

— , and Coauthors, 2013: Contribution of ocean overturning circulation to tropical rainfall peak in the Northern Hemisphere. Nat. Geosci., 6, 940-944, doi:10.1038/ngeo1987.

Haberle, R. M., J. B. Pollack, J. R. Barnes, R. W. Zurek, C. B. Leovy, J. R. Murphy, H. Lee, and J. Schaeffer, 1993: Mars atmospheric dynamics as simulated by the NASA Ames General Circulation Model: 1. The zonal-mean circulation. J. Geophys. Res, 98, 3093-3123, doi:10.1029/92JE02946.

Hartmann, D. L., 1994: Global Physical Climatology. International Geophysics Series, Vol. 56, Academic Press, 411 pp.
Held, I. M., and A. Y. Hou, 1980: Nonlinear axially symmetric circulations in a nearly inviscid atmosphere. J. Atmos. Sci., 37, 515-533, doi:10.1175/1520-0469(1980)037<0515: NASCIA $>2.0 . \mathrm{CO} ; 2$.

Janowiak, J. E., P. A. Arkin, P. Xie, M. L. Morrissey, and D. R. Legates, 1995: An examination of the east Pacific ITCZ rainfall distribution. J. Climate, 8, 2810-2823, doi:10.1175/ 1520-0442(1995)008<2810:AEOTEP > 2.0.CO;2.

Kang, S. M., I. M. Held, D. M. Frierson, and M. Zhao, 2008: The response of the ITCZ to extratropical thermal forcing: Idealized slab-ocean experiments with a GCM. J. Climate, 21, 35213532, doi:10.1175/2007JCLI2146.1.

— D. M. Frierson, and I. M. Held, 2009: The tropical response to extratropical thermal forcing in an idealized GCM: The importance of radiative feedbacks and convective parameterization. J. Atmos. Sci., 66, 2812-2827, doi:10.1175/2009JAS2924.1.

Kaspi, Y., and A. P. Showman, 2015: Atmospheric dynamics of terrestrial exoplanets over a wide range of orbital and atmospheric parameters. Astrophys. J., 804, 60, doi:10.1088/ 0004-637X/804/1/60.

Levine, X. J., and T. Schneider, 2011: Response of the Hadley circulation to climate change in an aquaplanet GCM coupled to a simple representation of ocean heat transport. J. Atmos. Sci., 68, 769-783, doi:10.1175/2010JAS3553.1.

, and - 2015: Baroclinic eddies and the extent of the Hadley circulation: An idealized GCM study. J. Atmos. Sci., 72, 2744-2761, doi:10.1175/JAS-D-14-0152.1.

Lewis, S. R., 2003: Modelling the Martian atmosphere. Astron. Geophys., 44, 4.06-4.14, doi:10.1046/j.1468-4004.2003.44406.x.

Lindzen, R. S., and S. Nigam, 1987: On the role of sea surface temperature gradients in forcing low-level winds and convergence in the tropics. J. Atmos. Sci., 44, 2418-2436, doi:10.1175/ 1520-0469(1987)044<2418:OTROSS >2.0.CO;2.

— , and A. V. Hou, 1988: Hadley circulations for zonally averaged heating centered off the equator. J. Atmos. Sci., 45, 2416-2427, doi:10.1175/1520-0469(1988)045<2416:HCFZAH>2.0.CO;2.

Merlis, T. M., T. Schneider, S. Bordoni, and I. Eisenman, 2013: Hadley circulation response to orbital precession. Part I: Aquaplanets. J. Climate, 26, 740-753, doi:10.1175/JCLI-D-11-00716.1.

Mitchell, J. L., and G. K. Vallis, 2010: The transition to superrotation in terrestrial atmospheres. J. Geophys. Res., 115, E12008, doi:10.1029/2010JE003587.

and J. M. Lora, 2016: The climate of Titan. Annu. Rev. Earth Planet. Sci., 44, 353-380, doi:10.1146/annurev-earth-060115-012428. R. T. Pierrehumbert, D. M. Frierson, and R. Caballero, 2006: The dynamics behind Titan's methane clouds. Proc. Natl. Acad. Sci. USA, 103, 18421-18426, doi:10.1073/ pnas.0605074103.

- G. K. Vallis, and S. F. Potter, 2014: Effects of the seasonal cycle on superrotation in planetary atmospheres. Astrophys. J., 787, 23, doi:10.1088/0004-637X/787/1/23.

Navarra, A., and G. Boccaletti, 2002: Numerical general circulation experiments of sensitivity to Earth rotation rate. Climate Dyn., 19, 467-483, doi:10.1007/s00382-002-0238-8.

Neelin, J. D., and I. M. Held, 1987: Modeling tropical convergence based on the moist static energy budget. Mon. Wea. Rev., 115, 3-12, doi:10.1175/1520-0493(1987)115<0003:MTCBOT >2.0.CO;2.

Nie, J., W. R. Boos, and Z. Kuang, 2010: Observational evaluation of a convective quasi-equilibrium view of monsoons. J. Climate, 23, 4416-4428, doi:10.1175/2010JCLI3505.1.

Pauluis, O., 2004: Boundary layer dynamics and cross-equatorial Hadley circulation. J. Atmos. Sci., 61, 1161-1173, doi:10.1175/ 1520-0469(2004)061<1161:BLDACH>2.0.CO;2. 
Pfeffer, R. L., 1981: Wave-mean flow interactions in the atmosphere. J. Atmos. Sci., 38, 1340-1359, doi:10.1175/ 1520-0469(1981)038<1340:WMFIIT>2.0.CO;2.

Pierrehumbert, R. T., 2005: Climate dynamics of a hard snowball Earth. J. Geophys. Res., 110, D01111, doi:10.1029/ 2004JD005162.

Plumb, R. A., and A. Y. Hou, 1992: The response of a zonally symmetric atmosphere to subtropical thermal forcing: Threshold behavior.J. Atmos. Sci., 49, 1790-1799, doi:10.1175/ 1520-0469(1992)049<1790:TROAZS > 2.0.CO;2.

Porco, C. C., and Coauthors, 2005: Imaging of Titan from the Cassini spacecraft. Nature, 434, 159-168, doi:10.1038/ nature 03436.

Privé, N. C., and R. A. Plumb, 2007: Monsoon dynamics with interactive forcing. Part I: Axisymmetric studies. J. Atmos. Sci., 64, 1417-1430, doi:10.1175/JAS3916.1.

Ramage, C., 1974: Structure of an oceanic near-equatorial trough deduced from research aircraft traverses. Mon. Wea. Rev., 102, 754-759, doi:10.1175/1520-0493(1974)102<0754: SOAONE $>2.0 . \mathrm{CO} ; 2$.

Raymond, D. J., 2000: Thermodynamic control of tropical rainfall. Quart. J. Roy. Meteor. Soc., 126, 889-898, doi:10.1002/ qj. 49712656406

Sadler, J. C., 1975: The monsoon circulation and cloudiness over the GATE area. Mon. Wea. Rev., 103, 369-387, doi:10.1175/ 1520-0493(1975)103<0369:TMCACO > 2.0.CO;2.

Satoh, M., 1994: Hadley circulations in radiative-convective equilibrium in an axially symmetric atmosphere. J. Atmos. Sci., 51, 1947-1968, doi:10.1175/1520-0469(1994)051<1947: HCIREI $>2.0 . \mathrm{CO} ; 2$.

Schneider, T., 2006: The general circulation of the atmosphere. Annu. Rev. Earth Planet. Sci., 34, 655-688, doi:10.1146/ annurev.earth.34.031405.125144.

— the seasonal cycle of a Hadley circulation and implications for monsoon dynamics. J. Atmos. Sci., 65, 915-934, doi:10.1175/ 2007JAS2415.1.

_ T. Bischoff, and G. H. Haug, 2014: Migrations and dynamics of the intertropical convergence zone. Nature, 513, 45-53, doi:10.1038/nature13636.

Shekhar, R., and W. R. Boos, 2016: Improving energybased estimates of monsoon location in the presence of proximal deserts. J. Climate, 29, 4741-4761, doi:10.1175/ JCLI-D-15-0747.1.
Sobel, A. H., and C. S. Bretherton, 2000: Modeling tropical precipitation in a single column. J. Climate, 13, 4378-4392, doi:10.1175/1520-0442(2000)013<4378:MTPIAS>2.0.CO;2.

— , and J. D. Neelin, 2006: The boundary layer contribution to intertropical convergence zones in the quasi-equilibrium tropical circulation model framework. Theor. Comput. Fluid Dyn., 20, 323-350, doi:10.1007/s00162-006-0033-y.

_- J. Nilsson, and L. M. Polvani, 2001: The weak temperature gradient approximation and balanced tropical moisture waves. J. Atmos. Sci., 58, 3650-3665, doi:10.1175/ 1520-0469(2001)058<3650:TWTGAA > 2.0.CO;2.

Toma, V. E., and P. J. Webster, 2010: Oscillations of the intertropical convergence zone and the genesis of easterly waves. Part I: Diagnostics and theory. Climate Dyn., 34, 587604, doi:10.1007/s00382-009-0584-x.

Tomas, R. A., and P. J. Webster, 1997: The role of inertial instability in determining the location and strength of near-equatorial convection. Quart. J. Roy. Meteor. Soc., 123, 1445-1482, doi:10.1002/qj.49712354202.

_ J. R. Holton, and P. J. Webster, 1999: The influence of crossequatorial pressure gradients on the location of nearequatorial convection. Quart. J. Roy. Meteor. Soc., 125, 1107-1127, doi:10.1002/qj.1999.49712555603.

Waliser, D. E., and C. Gautier, 1993: A satellite-derived climatology of the ITCZ. J. Climate, 6, 2162-2174, doi:10.1175/ 1520-0442(1993)006<2162:ASDCOT >2.0.CO;2.

_ , and R. C. Somerville, 1994: Preferred latitudes of the intertropical convergence zone. J. Atmos. Sci., 51, 1619-1639, doi:10.1175/1520-0469(1994)051<1619:PLOTIC > 2.0.CO;2.

Walker, C. C., and T. Schneider, 2005: Response of idealized Hadley circulations to seasonally varying heating. Geophys. Res. Lett., 32, L06813, doi:10.1029/2004GL022304.

— , and — 2006: Eddy influences on Hadley circulations: Simulations with an idealized GCM. J. Atmos. Sci., 63, 33333350, doi:10.1175/JAS3821.1.

Williams, G. P., 1988: The dynamical range of global circulations: Part I. Climate Dyn., 2, 205-260, doi:10.1007/BF01371320.

Xie, S.-P., 2004: The shape of continents, air-sea interaction, and the rising branch of the Hadley circulation. The Hadley Circulation: Present, Past and Future, H. F. Diaz and R. S. Bradley, Eds., Springer, 121-152.

Yihui, D., and J. C. Chan, 2005: The East Asian summer monsoon: An overview. Meteor. Atmos. Phys., 89, 117-142, doi:10.1007/ s00703-005-0125-z. 